\title{
VALORES QUE SE FOMENTAN EN LA ESCUELA UNIVERSITARIA DE CIENCIAS DE LA SALUD
}

\section{Values Promoted by the University School of Health Sciences}

\author{
*María del Carmen Maldonado, **Isis Stephanie Mejía, **Leslie Victoria Ulloa
}

\section{RESUMEN}

Los valores humanos orientan el comportamiento de la persona, exigen del individuo buena dosis de esfuerzo y valentía. Aquí concluyen las acciones educativas de diversas instituciones y organizaciones sociales, los medios de difusión masiva, y por supuesto la familia y la escuela. Objetivo: Identificar los valores fomentados dentro de las carreras del área de la salud según percepción de los estudiantes que asisten en el segundo periodo académico del año 2014. Pacientes y Métodos: Investigación de tipo mixta, diseño cualitativo de teoría fundamentada y sistemática de codificación abierta de tipo transversal, se utilizó una entrevista aplicada a 30 alumnos de Enfermería, Medicina y Odontología de la Universidad Nacional Autónoma de Honduras, 10 de cada una. Resultados: Los valores más fomentados por los estudiantes en orden de percepción fueron responsabilidad con un $17 \%$, compromiso $16 \%$ y valores éticos $14 \%$. Para los estudiantes los valores son "principios, disciplinas, acciones, comportamientos humanos que se inculcan en el seno familiar desde la infancia y se continúan fomentando en las instituciones" En las tres carreras los estudiantes expresaron que hay carencia de valores, estas deficien-

*Profesora del Departamento de Ciencias de la Enfermería de la Escuela Universitaria de Ciencias de la Salud (EUCS) de la Universidad Nacional Autónoma de Honduras del Valle de Sula (UNAH- VS).

*Estudiante de IV año, Carrera de enfermería. (EUCS UNAH-VS)

Dirigir correspondencia a: maricarmen0501@hotmail.com

Recibido: 20 de Febrero 2,015 Aprobado 18 de Julio 2,015 cias se ven reflejadas en las actitudes que presentan algunos estudiantes con sus mismos compañeros o durante la práctica en los centros asistenciales. Conclusión: Los estudiantes consideran que los valores son importantes para el desarrollo personal y el trato hacia los pacientes. A pesar de tener conocimiento sobre ellos se mostraron interesados en que se les diera más énfasis a estos durante las clases.

\section{PALABRAS CLAVE}

Enfermería, Medicina, Odontología, Valores.

\section{ABSTRACT}

Human values guide the behavior of the person, require the individual good deal of effort and courage. This concludes the educational activities of various institutions and social organizations, mass media, and of course the family and school. Objective: To identify the values promoted within the racing area of health perception as students attending the second academic period of 2014. Patients and Methods: descriptive transversal research, an interview applied to 30 students use Nursing, Medicine and Dentistry at the National Autonomous University of Honduras. Results: Most fostered by students in order of perceived values were $17 \%$ responsibility, commitment and ethical values $16 \% 14 \%$ For students values are "principles, disciplines, actions, human behaviors that are instilled within familiar from childhood and continue promoting insti- 
tutions "In the three races the students expressed that there is a lack of values, these shortcomings are reflected in the attitudes that have some students with their own peers or during practice in health centers. Conclusion: The values are considered by the very important students for personal development and treatment of patients, despite having knowledge about them were interested in being given more interest to these during lessons because of various deficiencies They were detected in some of them.

\section{KEYWORDS}

Nursery, Medicin, Odontology, Valores.

\section{INTRODUCCIÓN}

Los valores humanos orientan el comportamiento de la persona, exigen del individuo buena dosis de esfuerzo y valentía. El ser humano tiene vocación a pensar, a razonar, a medir las consecuencias de sus acciones y a correr con responsabilidades. Ahora bien la formación de valores en las nuevas generaciones depende en grado sumo de la continuidad histórica del proceso revolucionario.

Los valores son aquellos principios, normas o conductas que guían el comportamiento de las personas y cuya formación comienza desde el hogar, primeramente por enseñanza o por el ejemplo estando también influenciados por el entorno de cada individuo.(1) La formación del estudiante de las Ciencias Médicas, tiene que ser eminentemente moral, sin que esto signifique desconocer la preparación científica, y es responsabilidad de las instituciones de formación. Los profesionales del área de la salud se han caracterizado por la responsabilidad con la que atienden al ser humano en lo relativo a la salud. Cada paciente confía que el profesional hará lo necesario para resolver su problema de salud.
Los valores y principios pueden conformar actitudes o conductas predominantes en la praxis de las personas o colectivos. Todo esto es estudiado dentro de las éticas profesionales, enmarcando la ética médica, admitida también como bioética médica, bioética clínica o sanitaria. Siendo una de las más asociadas a las profesiones de la salud la bioética clínica que se ocupa del proceso de toma de decisiones en torno al paciente, e implica a los actores que intervienen en el mismo. ${ }^{(2)}$

Las distintas carreras de la salud cuentan con su propio enfoque de valores según la formación. La Carrera de Enfermería tiene como propósito, la formación de profesionales de enfermería que responda a las necesidades de salud de la población en su contexto, congruente a las nuevas metodologías, avances científico-técnicos y a la situación cambiante del país, sistema de salud y grupos poblacionales. ${ }^{(3)}$ La carrera de Odontología está encargada de formar profesionales capaces de dar respuesta a las necesidades de salud bucal del pueblo hondureño. ${ }^{(4)}$ La Carrera de Medicina tiene como finalidad formar profesionales médicos con visión integral, capaces de detectar problemas de salud individual y comunitaria, establecer diagnósticos e instaurar medidas preventivas, utilizando la tecnología en forma racional con el propósito de mejorar la calidad de vida de las comunidades y los problemas de salud individual. ${ }^{(5)}$ Los valores hacen referencia a modelos ideales de actuar y de existir que el ser humano aprecia, desea, busca y a través de los cuales interpreta el mundo y da significado a su existencia. Es necesario ver a la universidad como la generadora del potencial 
humano que se necesita para la transformación y desarrollo de la sociedad. ${ }^{(6)}$ Muchos podrían pensar que la universidad debería de enfocarse en la mera transmisión del conocimiento pero se ha destacado en diversas universidades la importancia de fomentar y reforzar los valores en el aula de clases como parte de una formación integral en los estudiantes lo cual en los años que lleven de estudio puedan adquirir actitudes con una marcada ética profesional.

El objetivo de este estudio fue identificar los valores fomentados dentro de las carreras del área de la salud según percepción de los estudiantes que asisten en el segundo periodo académico del año 2014.

\section{METODOLOGÍA}

Se realizó una investigación de tipo mixta, diseño cualitativo de teoría fundamentada sistemática de codificación abierta de tipo transversal, realizado a estudiantes de Enfermería, Medicina y Odontología en la Universidad Nacional Autónoma en el Valle de Sula en el segundo período académico del año 2014. La muestra fue no probabilística de 30 estudiantes de Medicina, Enfermería y Odontología, 10 de cada carrera, que se encontraban cursando del tercer año en adelante. Se elaboró una entrevista utilizando un cuestionario con preguntas abiertas, en aspectos cualitativos y cuantitativos. Los aspectos cualitativos se agruparon en las siguientes categorías:

Categoría 1. Qué son los valores.

Categoría 2. Importancia que tiene el fomento de los valores para los estudiantes.

Categoría 3. Cómo es la relación entre sus compañeros.
Categoría 4. Deficiencias que observa en el sistema de valores de los estudiantes de cada carrera.

Categoría 5. Frecuencia en que se brinda tiempo durante las clases para hablar sobre valores.

Categoría 6. Importancia que tienen los valores en el desarrollo personal y en el trato hacia los pacientes.

Categoría 7. Interés de los estudiantes en que se brinde más tiempo durante las clases para hablar sobre valores.

Los entrevistados dieron su consentimiento informado de forma verbal. El análisis de datos se hizo manual.

\section{RESULTADOS}

El aspecto cualitativo, fue estudiado en base a siete categorías, que se describen a continuación.

\section{Categoría 1. Qué son los valores.}

La opinión en general que tuvieron la mayoría de los estudiantes de las distintas carreras de la salud que fueron objeto de estudio sobre el significado de los valores fue la siguiente:

"Son principios, disciplinas, acciones, comportamientos humanos que se inculcan en el seno familiar desde la infancia y se continúan fomentando en las instituciones educativas, estos valores nos permiten desenvolvernos moralmente aplicándolos en el transcurso de la vida para convivir mejor y sanamente."

Categoría 2. Importancia que tiene el fomento de los valores para los estudiantes. Para los estudiantes entrevistados los valores:

"Son un componente esencial para mantener 
las buenas relaciones interpersonales" además consideran que el fomento de los mismos les ayudaría a dar un trato digno y humanizado a los pacientes, practicando de esta manera la ética y desarrollando sus carreras con profesionalismo."

\section{Categoría 3. Cómo es la relación entre sus} compañeros.

En esta categoría las opiniones de los estudiantes en las distintas carreras variaron:

Enfermería: "La relación es buena y cordial, básicamente laboral o por afinidad", "Por conveniencia y no hay compañerismo ni sinceridad, hay mucha rivalidad."

Odontología: "Las relaciones son buenas, hay amistad, compañerismo, unidad, solidaridad, respeto entre los mismos compañeros y pacientes."

Medicina: "La relación suele ser buena pero en algunos casos hay grupos divididos y cerrados de amistad", "las relaciones con los demás son muy superficiales, meramente profesional, solamente cuando es necesario trabajar en equipo" "las relaciones son malas y sin compañerismo."

\section{Categoría 4. Deficiencias que observa en} el sistema de valores de los estudiantes de cada carrera

Las distintas carreras cuentan con su propio sistema de valores con el objetivo de cumplir con las exigencias del medio en que se desempeñaran como profesionales, en cuanto a las deficiencias que existen, según las opiniones de los alumnos fueron:

Enfermería: "No hay promoción para hacer sobresalir la carrera, hay falta de solidaridad, de responsabilidad, respeto, liderazgo, compromiso y honestidad.

Odontología: "Falta compromiso, respeto, honestidad, solidaridad, tolerancia, responsabilidad, ética, liderazgo y compañerismo para un mejor trato a los pacientes y entre colegas"

Medicina: "Hay falta de empatía y mucha critica entre colegas y envidia, no hay respeto, solidaridad, honestidad, responsabilidad".

Categoría 5. Frecuencia en que se brinda tiempo durante las clases para hablar sobre valores.

En esta categoría todos los alumnos coincidieron en que el tema de valores es comentado en pocas ocasiones según la asignatura cursada," cuando se habla de los mismos es al inicio del periodo y antes de iniciar las práctica"

Categoría 6. Importancia que tienen los valores en el desarrollo personal y en el trato hacia los pacientes.

Los estudiantes de las tres carreras consideran de mucha importancia el desarrollo de los valores para brindar un mejor trato al paciente, y crecer como individuo y profesional.

Categoría 7. Interés de los estudiantes en que se brinde más tiempo durante las clases para hablar sobre valores.

Todos los entrevistados expresaron su interés en que el desarrollo de valores sea incluido en el tiempo de clases porque lo consideran fundamental en su formación especialmente por ser estudiantes de las carreras de la salud.

En la parte cuantitativa; los valores fomentados en las carreras de la salud de la UNAH-VS, son los siguientes: la responsabilidad $(17 \%)$, seguido del compromiso (16\%) y valores éticos (14\%). Ver Gráfica No 1. 
Gráfica No 1: Valores fomentados en las carreras de las ciencias de la salud. UNAH-VS.

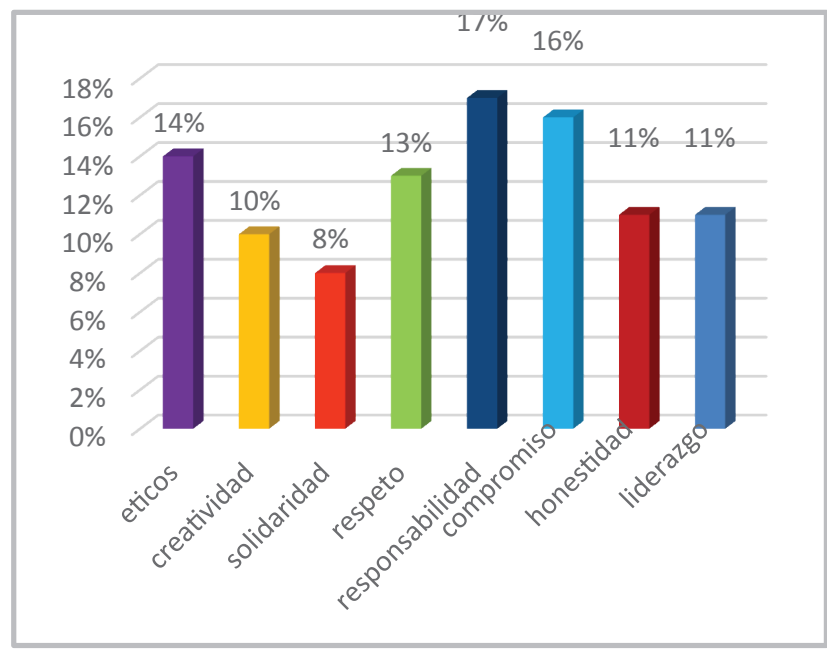

Fuente: Encuesta aplicada a los estudiantes de Enfermería, Medicina y Odontología, 2do periodo académico 2014.

\section{Discusión:}

En nuestra época la problemática de los valores ha adquirido relevancia de primer orden, y está en el centro de agudas discusiones teórico - filosófica y esto ha hecho que muchos autores, como el Dr. Juan Morí Lois exprese: "La educación debe priorizar los valores y revelar los ideales, preparar al hombre para la vida." ${ }^{(7)}$

Uno de los propósitos esenciales de nuestra educación es formar un hombre con principios y valores que les permitan enfrentar las complejas situaciones, asimilar los cambios y buscar soluciones acertadas a los problemas complejos del mundo moderno.

La calidad en la formación del profesional depende no sólo de los conocimientos y habilidades que desarrolle en el currículum universitario sino también de los intereses y valores que regulan su actuación profesional. Sin embargo en este estudio los estudiantes expresaron que los valores se inculcan en el seno familiar desde la infancia y se continúan fomentando en las instituciones educativas, esto coincide parcialmente con la opinión de los docentes de una institución en un estudio realizado en Cuba, donde el $41.3 \%$ de los profesores consideran que los valores se forman en edades tempranas por tanto no tiene sentido hablar de formación de valores en la Universidad. ${ }^{\left({ }^{(8)}\right.}$ En ese mismo estudio el 9,61\% no estuvieron de acuerdo con el criterios expresado por su colegas, pues consideran que la formación de valores es responsabilidad de todos los docentes universitarios. En este sentido las investigaciones demuestran que una motivación profesional sustentada en sólidos intereses profesionales puede actuar como elemento rector en el desarrollo de la personalidad del estudiante universitario y en particular en el desarrollo de valores morales y culturales asociados a la actuación profesional. $^{(9)}$

El amor a la profesión, la responsabilidad, la honestidad constituyen valores esenciales reguladores de la actuación de un profesional competente. ${ }^{(9)}$

En este estudio los estudiantes entrevistados reconocen la importancia de los valores para lograr una buena convivencia y promover las buenas relaciones interpersonales ya que como agentes de la salud se desenvuelven en un área donde deben trabajar en equipo con personas de distintas disciplinas y de manera integral, además identifican que el área de trabajo puede crear situaciones estresantes pero que esto no debe afectar el enfoque y principal objetivo que es la salud del paciente, así como brindarle un trato digno y humanizado. Esto coincide con lo expresado en este nuevo siglo por Julio 
Frenk quien sintetizó en tres, los grandes retos del Sistema Nacional de Salud: la protección financiera, la equidad y la calidad. El tercero de estos, la calidad, significa que la actuación de los trabajadores de la salud debe cumplir con requisitos técnicos pero también con buen trato al paciente y su familia y ello implica amabilidad, consideración y respeto a su dignidad humana y están expresadas las formas de cumplirse; lo más seguro es que la falta de estos atributos, es lo que genera la inconformidad de los pacientes al grado de denunciar, en algunos casos, ante la Comisión de Derechos Humanos. ${ }^{(10)}$

La inteligencia emocional se ha presentado como una característica que facilita las relaciones interpersonales, debido a que permite ser consciente de las emociones, comprenderlas, manejarlas en uno mismo y en otros, y utilizarlas para razonar mejor. Según las opiniones de los estudiantes de este estudio, las relaciones interpersonales se observan deficientes o limitadas, el trato es estrictamente necesario y se observa una clara falta de compañerismo en la mayoría de las respuestas, esto podría ser el resultado de relaciones superficiales que desencadena rivalidades y se caracterizan por una comunicación ineficiente y carencia de tolerancia. ${ }^{(11)}$

La deficiencia percibida de los valores como la solidaridad, honestidad, respeto y responsabilidad expresados por los estudiantes concuerda con la problemática que enfrentan pues influye negativamente en las relaciones interpersonales y en una adecuada actitud en el ejercicio de su profesión. Esto coincide con un estudio realizado en Venezuela donde existía una deficiencia en los valores interpersonales como son la solidaridad y el compañerismo. En Cuba sucedió algo similar pero es más marcada la deficiencia de valores como son el Humanismo, Amor al trabajo, Honestidad, Solidaridad y Respeto. ${ }^{(12)}$

Se determinó que durante las clases si se habla de valores pero que esto se da generalmente al inicio de las clases y antes de iniciar las diferentes prácticas, en algunos momentos los docentes buscan la manera de interrelacionar las temáticas tratadas con la importancia de los distintos valores.

Para nadie es un secreto que el docente universitario es un especialista en su profesión pero carece de formación psicopedagógica. Esta es generalmente adquirida a través de la educación de postgrado y no siempre de forma sistemática. Por tanto es imprescindible que el docente universitario reciba la preparación psicopedagógica necesaria para diseñar, ejecutar y dirigir un proceso de enseñanza aprendizaje que propicia la educación de valores teniendo en cuenta que este es un proceso dialógico, participativo en el que el docente y estudiantes asumen la condición de sujetos de enseñanza y aprendizaje. ${ }^{(13)}$

Los estudiantes afirmaron que los valores son imprescindibles para brindar un trato digno y humanizado al paciente y también aceptan que la aplicación de los mismos les permitirá crecer y desarrollarse en todos los ámbitos, fortaleciendo además las relaciones interpersonales.

Los valores más fomentados por los estudiantes según este estudio en orden de 
percepción fueron responsabilidad con un $17 \%$, compromiso $16 \%$ y valores éticos $14 \%$, esto es similar a otros estudios donde se hace énfasis en valores como autonomía, responsabilidad, orden, coherencia, eficacia y reflexividad. ${ }^{(14)}$

\section{CONCLUSIONES}

El docente universitario debe ser un modelo educativo para sus estudiantes. En la medida que el docente exprese en su actuación profesional y en sus relaciones con los estudiantes valores tales como la responsabilidad, el amor a la patria y a la profesión, la honestidad, la justicia entre otros propiciará su formación como motivo de actuación en los estudiantes.

Sólo creando espacios de reflexión en el proceso de enseñanza-aprendizaje en los que el estudiante aprenda a valorar, argumentar sus puntos de vista, defenderlos ante los que se oponen a ellos, en los que el estudiante tenga libertad para expresar sus criterios, para discrepar, para plantear iniciativas, para escuchar y comprender a los demás, para enfrentarse a problemas con seguridad e independencia, para esforzarse por lograr sus propósitos, espacios en el proceso de enseñanza-aprendizaje en los que sean los docentes universitarios guías de sus estudiantes, modelos de profesionales, ejemplos a imitar, sólo en estas condiciones estaremos contribuyendo a la educación de valores del estudiante universitario.

Tendríamos que preguntarnos los docentes universitarios. ¿Estamos preparados para enfrentar este reto?

\section{RECOMENDACIONES}

1.- Comprender que la las carreras del área de la salud son carreras humanistas, que aplican el conocimiento técnico científico para desenvolverse en diversas áreas en las cuales los valores son indispensables para llevar a cabo las acciones de acuerdo a lo moralmente correcto y según lo establecido por la ley.

2.- Fomentar y fortalecer en la academia los valores éticos y morales tanto los docentes como los estudiantes, evitando así la doble moral.

\section{BIBLIOGRAFÍA}

1. Amaro C, Andino L, Flores O, Ruiz S, Coello C, Sabillon M. et al. Ética para la Construcción de la Ciudadanía. Tegucigalpa. Industrias Gráficas INGRAE. 2013.

2. Sorensen A. "Justice and Democracy. Some preliminary reflections." In Francisco Javier López Frias. Bioética, neuroética, libertad y justicia Granada: Comares; 2013. pp 494-508.
3. Vicerrectoría de Orientación. Asuntos Estudiantiles VOAE Ciudad Universitaria "José Trinidad Reyes". Perfil de Estudios de la carrera de Enfermería Código 10; 2009.

4. Vicerrectoría de Orientación y Asuntos Estudiantiles VOAE Ciudad Universitaria "José Trinidad Reyes". Perfil de Estudios de la carrera de Odontología Código 09; 2009. 
5. "Vicerrectoría de Orientación y Asuntos Estudiantiles VOAE Ciudad Universitaria "José Trinidad Reyes". Perfil de Estudios de la carrera de Medicina Código 07; 2009 .

6. Pestarino E. La ética Profesional [Internet] Bogotá: Crowe Horthwath International; 2011. [4 Octubre 2014]. Disponible https://es.scribd.com/doc/.../EticaProfesional-Eduardo-Pestarino

7. Aguilar Díaz, Cándido. Fortalecimiento de valores: Una necesidad de todos los tiempos. - Camagüey: ISPJM, 1998. Leer más: http://www.monografias.com/ trabajos14/valores-estud/valores-estud. shtml\#ixzz3hKkx4sRu

8. González Maura Viviana. La Educación de Valores en el Curriculum Universitario. Un enfoque Psicopedagogico para su estudio. Rev Cubana Educ Med Super 2000; 14(1):74-82.

9. Febles M. Caracterización psicológica del estudiante de nuevo ingreso y su seguimiento. Tesis de Maestría en Psicología Educativa. Facultad de Psicología. Universidad de La Habana. 1997.

10. Morales González JA, Nava CG, Esquivel SJ, Díaz PLE. Principios de ética y bioética y conocimiento del hombre. [Internet] Hidalgo México: Abasolo;
2011. Disponible.www.uaeh.edu.mx/investigacion/.../4822/libro_principios_ de_etica.pdfde G Nava Chapa - 2011.

11. Llano G. Castillo O, Sánchez E, Lorenzo M, Díaz V. Formación de Valores en estudiantes. Papel de los profesionales de la información. Gaceta Medica Espirituana. 2007;9(2). http://bvs.sld.cu/re vistas/gme/pub/vol.9.\%282\%29_19/ p19.html.

12. Capote Eduardo, Villegas Hyxia, Brett María Alejandra, Guada Natacha, Capote José. Comparación de la jerarquía de valores entre los estudiantes de medicina y odontología del segundo año de la universidad de Carabobo. Acta Odontológica Venezolana. 2006[citado 13 junio 2015];44(3) Disponible en: http://www.actaodontologica.com/ ediciones/2006/3/jerarquia_valores estudiantes_medicina_odontologia.asp

13. Ojalvo V. Concepción de la enseñanza aprendizaje y organización docente para la formación de valores de estudiantes universitarios. Diseño de investigación. Ciudad de la Habana. CEPES. 1997.

14. Hoyos G, Martínez MM. Que significa educar en valores Hoy. [Internet] Barcelona: Octaedro; 2004 [citado 5 de Octubre 20014] www.octaedro.com/OCTart. asp? ...significa+educar...valores+hoy\% $3 \mathrm{~F}$. 